\title{
THE $\aleph_{2}$-SOUSLIN HYPOTHESIS
}

BY

\author{
RICHARD LAVER $^{1}$ AND SAHARON SHELAH ${ }^{2}$
}

Abstract. We prove the consistency with $C H$ that there are no $\aleph_{2}$-Souslin trees.

The $\boldsymbol{\aleph}_{2}$-Souslin hypothesis, $\boldsymbol{S H}_{\boldsymbol{*}_{2}}$, is the statement that there are no $\boldsymbol{\aleph}_{2}$-Souslin trees. In Mitchell's model [5] from a weakly compact the stronger statement holds (Mitchell and Silver) that there are no $\aleph_{2}$-Aronszajn trees, a property which implies that $2^{\kappa_{0}}>\aleph_{1}$.

THEOREM. Con $(Z F C+$ there is a weakly compact cardinal $)$ implies

$$
\operatorname{Con}\left(Z F C+2^{\kappa_{0}}=\kappa_{1}+S H_{*_{2}}\right) \text {. }
$$

In the forcing extension, $2^{\kappa_{1}}$ is greater than $\boldsymbol{\kappa}_{2}$, and can be arbitrarily large. Analogues of this theorem hold with $\aleph_{2}$ replaced by the successor of an arbitrary regular cardinal. Strengthenings and problems are given at the end of the paper.

Let $\mathfrak{N}$ be a ground model in which $\kappa$ is a weakly compact cardinal. The extension which models $\mathrm{SH}_{\boldsymbol{\kappa}_{2}}$ and $\mathrm{CH}$ is obtained by iteratively forcing $>\kappa^{+}$times with certain $\kappa c c$, countably closed partial orders, taking countable supports in the iteration. For $\alpha \geqslant 1,\left(\mathscr{P}_{\alpha}, \leqslant\right)$ is the ordering giving the first $\alpha$ steps in the iteration. $\mathcal{P}_{\alpha}$ is a set of functions with domain $\alpha$.

Let $L_{\kappa_{1} \kappa}$ be the Levy collapse by countable conditions of each $\beta \in\left[\kappa_{1}, \kappa\right)$ to $\kappa_{1}$ (so $\kappa$ is the new $\kappa_{2}$ ). Then $\mathscr{P}_{1}$ (isomorphic to $L_{\kappa_{1} \kappa}$ ) is $\left\{f: \operatorname{dom} f=1, f(0) \in L_{\kappa_{1} \kappa}\right\}$, ordered by $f \leqslant g$ iff $f(0) \leqslant g(0)$. To define $\mathscr{P}_{\beta+1}$, choose a term $A_{\beta}$ in the forcing language of $\mathcal{P}_{\beta}$ for a countably closed partial ordering (to be described later) and let $\mathscr{P}_{\beta+1}=\left\{f: \operatorname{dom} f=\beta+1, f \uparrow \beta \in \mathscr{P}_{\beta}, f \uparrow \beta H_{\mathscr{P}_{\beta}} f(\beta) \in A_{\beta}\right\}$, ordered by $f \leqslant g$ iff $f \uparrow \beta \leqslant g \uparrow \beta$ and $g \uparrow \beta H_{\mathscr{P}_{\beta}} f(\beta) \leqslant g(\beta)$. For $\alpha$ a limit ordinal, $\mathscr{P}_{\alpha}=\{f$ : $\operatorname{dom} f=\alpha, f \uparrow \beta \in \mathcal{P}_{\beta}$ for all $\beta<\alpha$, and $f(\beta)$ is (the term for) $\varnothing$, the least element of $A_{\beta}$, for all but $\leqslant \aleph_{0} \beta$ 's , ordered by $f \leqslant g$ iff for all $\beta<\alpha, f \uparrow \beta<g$ | $\beta$.

Each $\mathscr{P}_{\alpha}$ is countably closed. We are done as in Solovay-Tennenbaum [7] if the $A_{\beta}$ 's can be chosen so that each $\mathscr{P}_{\alpha}$ has the $\kappa c c$, and therefore that every $\boldsymbol{\aleph}_{2}$ ( $=\kappa)$-Souslin tree which crops up gets killed by some $A_{\beta}$.

If $T$ is a tree then $(T)_{\lambda}$ is the $\lambda$ th level of $T,(T)_{<\lambda}=\cup_{\mu<\lambda} T_{\mu}$. Regarding the previous problem, it is a theorem of Mitchell that if $C H$ and $\diamond\left\{\alpha<\omega_{2}: c f(\alpha)=\right.$ $\left.\aleph_{1}\right\}$ hold, then there are countably closed $\aleph_{2}$-Souslin trees $T_{n}, n<\omega$, such that for

Received by the editors October 18, 1978.

1980 Mathematics Subject Classification. Primary 02K35, 04A20.

'Supported by NSF grant MCS-76-06942.

${ }^{2}$ Supported by NSF grant MCS-76-08479. 
each $m<\omega, \bigotimes_{n<m} T_{n}$ has the $\aleph_{2} c c$, but $\bigotimes_{n<\omega} T_{n}$ does not have the $\aleph_{2} c c$. We give for interest his proof modulo the usual Jensen methods. At stage $\mu<\omega_{2}$ construct each $\left(T_{n}\right)_{\mu}$ normally above $\left(T_{n}\right)_{<\mu}$. If $\mu=\nu+1$ let each $x \in\left(T_{n}\right)_{\nu}$ have at least two successors in $\left(T_{n}\right)_{\mu}$. If $c f(\mu)=\omega$ let all branches in $\left(T_{n}\right)_{<\mu}$ go through. If $c f(\mu)=\omega_{1}$ make sure that the antichain given by the $\diamond$-sequence for $\otimes_{n<m_{\mu}} T_{n}$ is taken care of, and choose $\left\langle c_{\mu n}: n<\omega\right\rangle \in \otimes_{n<\omega}\left(T_{n}\right)_{\mu}$ so that if $\mu^{\prime}<\mu, c f\left(\mu^{\prime}\right)=$ $\omega_{1}$, then $\left\langle c_{\mu^{\prime} n}: n\langle\omega\rangle \nless\left\langle c_{\mu n}: n\langle\omega\rangle\right.\right.$. We also carry along the following induction hypothesis: if $\nu<\mu,\left\langle x_{n}: n<\omega\right\rangle \in \bigotimes_{n<\omega}\left(T_{n}\right)_{v}, \quad m<\omega, \quad\left\langle y_{n}: \quad n<m\right\rangle \in$ $\bigotimes_{n<m}\left(T_{n}\right)_{\mu}, x_{n}<y_{n}(n<m)$ and $\left\langle x_{n}: n<\omega\right\rangle\left\langle c_{\lambda n}: n\langle\omega\rangle\right.$, for all $\lambda \leqslant \nu$ with $c f(\lambda)=\omega_{1}$, then there are $y_{n} \in\left(T_{n}\right)_{\mu}(m \leqslant n<\omega)$ with $x_{n}<y_{n}$, such that $\left\langle y_{n}\right.$ : $n\langle\omega\rangle \neq\left\langle c_{\lambda n}: n\langle\omega\rangle\right.$, for all $\lambda \leqslant \mu$ with $c f(\lambda)=\omega_{1}$.

If $\delta$ is inaccessible, then forcing with $L_{\kappa_{1} \delta}$ (whence $2^{\kappa_{0}}=\kappa_{1}, 2^{\kappa_{1}}=\kappa_{2}=\delta$, and $\diamond\left\{\alpha<\omega_{2}: c f(\alpha)=\omega_{1}\right\}$ hold) followed by forcing with the $\otimes_{n<\omega} T_{n}$ constructed previously, gives a countably closed length $\omega$ iteration of countably closed, $\delta c c$ partial orderings which does not have $\delta c c$.

The previous theorem does not rule out that an iteration of $\boldsymbol{\aleph}_{2}$-Souslin trees can give $\mathrm{CH}$ and $\mathrm{SH}_{\kappa_{2}}$; in this paper, though, the $\kappa_{2}$-Souslin trees are killed by a different method. Let $T$ be an $\boldsymbol{\kappa}_{2}$-Souslin tree (we may assume without loss of generality that $T$ is normal and $\left.\operatorname{Card}(T)_{1}=\aleph_{1}\right)$. The antichain partial order $A_{T}$ is defined to be ( $\{x \subseteq T: x$ a countable antichain, root $T \notin x\}, \subseteq$ ). Now $A_{T}$ need not have the $\aleph_{2} c c$, as shown by the following result of the first author: $\operatorname{Con}(Z F C)$ implies $\operatorname{Con}\left(Z F C+\right.$ "there is an $\boldsymbol{\aleph}_{2}$-Souslin tree $T$ and a sequence $\left\langle d_{\alpha n}: n\langle\omega\rangle\right.$ from $(T)_{\alpha}$, for each $\alpha<\omega_{2}$, such that if $\alpha<\beta$, there is an $m<\omega$ with $d_{\alpha n}<d_{\beta n}$, for all $n>m$ ".) Namely, start with a model of $C H$. Determine in advance that, say, $(T)_{\alpha}=\left[\omega_{1} \alpha, \omega_{1}(\alpha+1)\right)$ and that $d_{\alpha n}=\omega_{1} \alpha+n$. Conditions are countable subtrees $S$ of $T$ such that if $S \cap(T)_{\alpha} \neq \varnothing$ then $\left\{d_{\alpha n}: n<\omega\right\} \subseteq S$, which meet the requirements on the $d_{\beta n}$ 's.

Devlin [2] has shown that such a tree exists in $L$.

We show now that if each $A_{\beta}$ is an $A_{T}, T$ an $\aleph_{2}$-Souslin tree, then each $\mathscr{P}_{\alpha}$ has the $\kappa c c$, which will prove the theorem (we actually just use that Card $T<$ the cardinal designated as the new $2^{k_{1}}$ and $T$ has no $\omega_{2}$-paths; see remarks at the end). This theorem was originally proved by the first author when $\kappa$ is measurable; that the assumption can be weakened to weak compactness of $\kappa$ is due to the second author.

We consider now only the case $\alpha \leqslant \kappa^{+}$(which will suffice, assuming $2^{\kappa}=\kappa^{+}$in $\Re$, for $C H+S H_{\kappa_{2}}+2^{\kappa_{1}}=\kappa_{3}$ ); $\alpha$ arbitrary will be dealt with at the end.

Fix $\alpha$ for the rest of the proof. We assume by induction that

(1) For each $\beta<\alpha, \mathscr{P}_{\beta}$ has the $\kappa c c$.

(One more induction hypothesis is listed later.)

For $\beta<\alpha$, let $T_{\beta}$ be the $\beta$ th $\kappa_{2}$-Souslin tree, so $\mathscr{P}_{\beta+1}=\mathscr{P}_{\beta} \tilde{\otimes} A_{\beta}$, where $A_{\beta}=A_{T_{\beta}}$. Assume without loss of generality that for each $\lambda<\kappa$,

$$
\left(T_{\beta}\right)_{\lambda} \subseteq\left[\omega_{1} \lambda, \omega_{1}(\lambda+1)\right) \text {. }
$$

An $f \in \mathscr{P}_{\beta}, \beta \leqslant \alpha$, is said to be determined if there is in $\mathscr{N}$ a sequence $\left\langle z_{\gamma}\right.$ : $\gamma \in \operatorname{dom} f-\{0\}>$ of countable sets of ordinals such that for all $\gamma \in \operatorname{dom} f-\{0\}$, 
$f \nmid \gamma H_{\Phi_{\gamma}} f(\gamma)=z_{\gamma}$. If $\left\langle f_{n}: n\langle\omega\rangle\right.$ is a sequence of determined members of $\mathscr{P}_{\beta}$, with $f_{n} \leqslant f_{n+1}$, then the coordinatewise union $f_{\omega}$ of the $f_{n}$ 's is seen to be a determined member of $\mathscr{P}_{\beta}$ extending each $f_{n}$. From this it may be seen, by induction on $\beta \leqslant \alpha$, that the set of determined members of $\mathscr{P}_{\beta}$ is cofinal in $\mathscr{P}_{\beta}$. Redefine each $\mathscr{P}_{\beta}$ then to consist just of the determined conditions. Clearly Card $\mathscr{P}_{\beta} \leqslant \kappa$, for all $\beta \leqslant \alpha$.

For $f, g \in \mathscr{P}_{\beta}, f \sim g$ means that $f$ and $g$ are compatible.

Fix for the rest of the proof a one-one enumeration $\alpha=\left\{\alpha_{\mu}: \mu \in S\right\}$, for some $S \subseteq \kappa$ (this induces a similar enumeration of each $\beta<\alpha$, the induction hypothesis (2) for $\beta$ below, is with respect to this induced enumeration). For notational simplicity we now assume that $S$ is some $\kappa^{\prime}<\kappa$.

If $\lambda<\kappa, \beta \leqslant \alpha, f \in \mathscr{P}_{\beta}$, define $f \mid \lambda$ to be the function $h$ with domain $\beta$ such that $h(\gamma)=\varnothing$ unless $\gamma \in\left\{\alpha_{\mu}: \mu<\lambda\right\} \cap \beta$, in which case,

$$
\gamma=0 \Rightarrow h(\gamma)=f(\gamma) \uparrow\left(\omega_{1} \times \lambda\right), \quad \gamma>0 \Rightarrow h(\gamma)=f(\gamma) \cap \lambda .
$$

The function $f \mid \lambda$ need not be a condition, but for $g \in \mathscr{P}_{\beta}$, we will still write $f \mid \lambda \leqslant g$ to mean that $f \mid \lambda$ is coordinatewise a subset of $g$. Let $\mathscr{P}_{\beta} \mid \lambda=\left\{f \in \mathscr{P}_{\beta}\right.$ : $f \mid \lambda=f\}$.

Suppose $0<\beta \leqslant \alpha, \lambda<\kappa$. Define

$$
\#_{\lambda}^{\beta}(f, g, h) \Leftrightarrow f, g \in \mathscr{P}_{\beta}, f|\lambda=g| \lambda=h,
$$

${ }_{\lambda}^{\beta}(f, h) \Leftrightarrow f \in \mathscr{P}_{\beta}, h \in \mathscr{P}_{\beta} \mid \lambda$ and for every $h^{\prime} \geqslant h$ with $h^{\prime} \in \mathscr{P}_{\beta} \mid \lambda, h^{\prime} \sim f$,

$$
*_{\lambda}^{\beta}(f, g, h) \Leftrightarrow *_{\lambda}^{\beta}(f, h) \text { and } *_{\lambda}^{\beta}(g, h) .
$$

For $P \subseteq Q, Q$ a partial ordering, $P \subseteq$ reg $Q$ means that $P$ is a regular subordering of $Q$, that is, any two members of $P$ compatible in $Q$ are compatible in $P$, and every maximal antichain of $P$ is a maximal antichain of $Q$. If $\mathscr{P}_{\beta} \mid \lambda \subseteq_{\mathrm{reg}} \mathcal{P}_{\beta}$, then $*_{\lambda}^{\beta}(f, h)$ states that $h H_{\Phi_{\beta} \mid \lambda} \llbracket f \rrbracket \neq 0$.

Recall that the sets of the form $\left\{\lambda<\kappa:\left(R_{\lambda}, \in, A \cap R_{\lambda}\right) \vDash \Phi\right\}$, where $A \subseteq \kappa, \Phi$ is $\pi_{1}^{1}$, and $\left(R_{\kappa}, \in, A\right) \vDash \Phi$, belong to a normal uniform filter $\mathscr{F}_{w c}$, the weakly compact filter on $\kappa$ (see [9], [0]). The second thing we assume by induction is

(2) for all $\beta<\alpha$, for $\mathscr{F}_{w c}$-almost all $\lambda<\kappa$, for all $f, g, h, \#_{\lambda}^{\beta}(f, g, h)$ implies that for some $h^{\prime} \geqslant h, *_{\lambda}^{\beta}\left(f, g, h^{\prime}\right)$.

If $\beta<\alpha, \lambda<\kappa$, say that $\left(T_{\beta}\right)_{<\lambda}$ is determined by $\mathscr{P}_{\beta} \mid \lambda$ if for each $\theta, \tau$ in $\left(T_{\beta}\right)_{<\lambda}$ there is a $\mathscr{P}_{\beta}$-maximal antichain $R$ of conditions deciding the ordering between $\theta$ and $\tau$ in $T_{\beta}$, such that $R \subseteq \mathscr{P}_{\beta} \mid \lambda$.

Lemma 1. There is a closed unbounded set of $\lambda<\kappa$ such that for all $\mu<\lambda,\left(T_{\alpha_{\mu}}\right)_{<\lambda}$ is determined by $\mathscr{P}_{\alpha_{\mu}} \mid \lambda$.

Proof. This is a consequence of the strong inaccessibility of $\kappa$ and the assumption that each $\mathscr{P}_{\beta}, \beta<\alpha$, has $\kappa c c$. 
LeMma 2. For $\mathscr{F}_{\text {wc }}$-almost all $\lambda<\kappa$,

(a) $\lambda$ is strongly inaccessible.

(b) For all $\mu<\lambda, \mathscr{P}_{\alpha_{\mu}} \mid \lambda$ has the $\lambda c c$.

(c) For all $\mu<\lambda, \mathscr{P}_{\alpha_{\mu}} \mid \lambda \subseteq \subseteq_{\text {reg }} \mathscr{P}_{\alpha_{\mu}}$.

(d) For all $\mu<\lambda, \mathbb{H}_{\mathscr{P}_{\alpha_{\mu}} \mid \lambda} \lambda=\boldsymbol{\kappa}_{2}$.

(e) For all $\mu<\lambda, H_{\mathcal{P}_{\alpha_{\mu}} \mid \lambda}\left(T_{\alpha_{\mu}}\right)_{<\lambda}$ is an $\boldsymbol{\kappa}_{2}$-Souslin tree.

Proof. By $\pi_{1}^{1}$ reflection and the normality of $\mathscr{F}_{w c}$.

LeMMA 3. Let $\beta \leqslant \alpha, \lambda<\kappa, \mathscr{P}_{\beta} \mid \lambda \subseteq{ }_{\mathrm{reg}} \mathscr{P}_{\beta}$.

(a) If $f \in \mathscr{P}_{\beta}, j \in \mathscr{P}_{\beta} \mid \lambda$, and $f \sim j$, then there is an $h \geqslant j$ with $*_{\lambda}^{\beta}(f, h)$.

(b) If $*_{\lambda}^{\beta}(f, g, h)$ and $D, E$ are cofinal subsets of $\mathscr{P}_{\beta}$, then there exists $\left\langle f^{\prime}, g^{\prime}, h^{\prime}\right\rangle$ $\geqslant\langle f, g, h\rangle$ with $*_{\lambda}^{\beta}\left(f^{\prime}, g^{\prime}, h^{\prime}\right), f^{\prime} \in D, g^{\prime} \in E, h \leqslant f^{\prime}, g^{\prime}$.

Proof. These are standard facts about forcing.

The following is T. Carlson's version of the lemma we originally used here.

Lemma 4. Suppose $\lambda$ satisfies Lemma 1 and Lemma $2(\mathrm{c}), \mu<\lambda$, and $*_{\lambda}^{\alpha_{\mu}}(f, h)$. Then $f \mid \lambda \leqslant h$.

Proof. Otherwise there is a $\nu<\lambda$ with $\alpha_{\nu}<\alpha_{\mu}$, and a $\theta \in f\left(\alpha_{\nu}\right) \cap \lambda$ such that $\theta \notin h\left(\alpha_{\nu}\right)$. We have that $h \uparrow \alpha_{\nu} H_{\Phi_{\alpha_{\nu}} \lambda} \theta$ is $T_{\alpha_{\nu}}$-incomparable with each member of $h\left(\alpha_{\nu}\right)$; otherwise $*_{\lambda}^{\alpha_{\mu}}(f, h)$ would be contradicted. Pick an $h^{\prime} \in \mathscr{P}_{\alpha_{\nu}} \mid \lambda, h^{\prime} \geqslant h \uparrow \alpha_{\nu}$, and a $\theta^{\prime}<\lambda$ such that $h^{\prime} H_{\Phi_{\alpha_{p}} \lambda} \theta<T_{\alpha_{v}} \theta^{\prime}$. Let $\bar{h}$ be $h^{\prime}\left\langle\left\langle\left(\alpha_{\nu}\right) \cup\left\{\theta^{\prime}\right\}\right\rangle-\right.$ $h \uparrow\left[\alpha_{\nu}+1, \alpha_{\mu}\right)$. Then $\bar{h} \in \mathscr{P}_{\alpha_{\mu}} \mid \lambda, h \leqslant \bar{h}$, and $\bar{h} \nsim f$, a contradiction.

Lemma 5. Suppose $\lambda$ satisfies Lemma 1 and Lemma $2(\mathrm{c}), \mu<\lambda$, and $*_{\lambda^{\alpha}}^{\alpha}(f, g, h)$. Then there is an $\left(f^{\prime}, g^{\prime}, h^{\prime}\right) \geqslant(f, g, h)$ with $\#_{\lambda}^{\alpha_{h}}\left(f^{\prime}, g^{\prime}, h^{\prime}\right)$.

Proof. Choose $(f, g, h)=\left(f_{0}, g_{0}, h_{0}\right) \leqslant \cdots \leqslant\left(f_{n}, g_{n}, h_{n}\right) \leqslant \cdots$ so that $*_{\lambda}^{\alpha} \alpha_{n}\left(f_{n}, g_{n}, h_{n}\right), h_{n} \leqslant f_{n+1}, h_{n} \leqslant g_{n+1}$. This is done by repeated applications of Lemma 3(a), (b). Then Lemma 4 implies that the coordinatewise union $\left(f^{\prime}, g^{\prime}, h^{\prime}\right)$ of the $\left(f_{n}, g_{n}, h_{n}\right)$ 's is as desired.

Definition. Suppose $\lambda<\kappa, \mu<\lambda, f, g \in \mathscr{P}_{\alpha_{\mu}}$, and suppose $\theta, \tau$ are nodes of $\left(T_{\alpha_{\mu}}\right)_{\geqslant \lambda}(\theta=\tau$ allowed). Then $\langle f, g\rangle$ is said to $\lambda$-separate $\langle\theta, \tau\rangle$ if there is a $\gamma\langle\lambda$ and $\theta^{\prime}, \tau^{\prime} \in\left(T_{\alpha_{\mu}}\right)_{\gamma}$, with $\theta^{\prime} \neq \tau^{\prime}$, such that

$$
f H_{\mathscr{P}_{\alpha_{\mu}}} \theta^{\prime}<T_{\alpha_{\mu}} \theta, \quad g H_{\mathscr{P}_{\alpha_{\mu}}} \tau^{\prime}<T_{\boldsymbol{\alpha}_{\mu}} \tau .
$$

Lemma 6. Suppose $\lambda$ satisfies Lemmas 1 and $2, \mu<\lambda, *_{\lambda}^{\alpha^{\alpha}}(f, g, h),\{\theta, \tau\} \subseteq$ $\left(T_{\alpha_{\mu}}\right)_{\geqslant \lambda}$, with $\theta=\tau$ allowed. Then there is an $\left.\left\langle f^{\prime}, g^{\prime}, h^{\prime}\right\rangle\right\rangle\langle f, g, h\rangle$ such that $*_{\lambda}^{\alpha_{\mu}}\left(f^{\prime}, g^{\prime}, h^{\prime}\right)$ and $\left\langle f^{\prime}, g^{\prime}\right\rangle \lambda$-separates $\langle\theta, \tau\rangle$.

Proof.

Claim. There are $f_{0}, f_{1} \geqslant f, \bar{h} \geqslant h$, with $*_{\lambda}^{\alpha_{\mu}}\left(f_{0}, \bar{h}\right), *_{\lambda}^{\alpha^{\alpha_{n}}}\left(f_{1}, \bar{h}\right)$, such that $\left\langle f_{0}, f_{1}\right\rangle$ $\lambda$-separates $\langle\theta, \theta\rangle$ via a $\left\langle\theta_{1}, \theta_{2}\right\rangle \in\left(T_{\alpha_{\mu}}\right)_{\gamma}$, for some $\gamma<\lambda$.

Proof. Consider the result of taking a generic set $G_{\alpha_{\mu}} \mid \lambda$ over $\mathscr{P}_{\alpha_{\mu}} \mid \lambda$ which contains $h$. In $\mathscr{N}\left[G_{\alpha_{\mu}} \mid \lambda\right],\left(T_{\alpha_{\mu}}\right)_{<\lambda}$ is a $\lambda\left(=\aleph_{2}\right)$-Souslin tree. In the further extension $\mathfrak{N}\left[G_{\alpha_{\mu}}\right], \theta$ determines a $\lambda$-path through $\left(T_{\alpha_{\mu}}\right)_{<\lambda}$. Since this path is not in $\mathfrak{N}\left[G_{\alpha_{\mu}} \mid \lambda\right]$, there must be $\bar{h} \in G_{\alpha_{\mu}} \mid \lambda, \bar{h} \geqslant h, f_{0}, f_{1} \geqslant f, \gamma<\lambda, \theta_{0}, \theta_{1} \in\left(T_{\alpha_{\mu}}\right)_{\gamma}, \theta_{0} \neq \theta_{1}$, with 
$\bar{h} H f_{0} H \theta_{0}<T_{\alpha_{\mu}} \theta, \bar{h} H f_{1} H \theta_{1}<T_{\alpha_{\mu}} \theta$, such that $*_{\lambda}^{\alpha_{\mu}}\left(f_{0}, \bar{h}\right)$ and $*_{\lambda}^{\alpha_{\mu}}\left(f_{1}, \bar{h}\right)$. This gives the claim.

Now, by Lemma 3, choose $\left(g^{\prime}, h^{\prime}\right) \geqslant(g, h)$ and a $\tau^{\prime} \in\left(T_{\alpha_{\mu}}\right)_{\gamma}$ so that $*_{\lambda^{\prime}}^{\alpha}\left(g^{\prime}, h^{\prime}\right)$ and $g^{\prime} \Vdash \tau^{\prime}<T_{a_{\mu}} \tau$. Pick $i \in\{0,1\}$ with $\tau^{\prime} \neq \theta_{i}$. Let $f^{\prime}=f_{i}, \theta^{\prime}=\theta_{i}$. Then $\left(f^{\prime}, g^{\prime}, h^{\prime}\right)$ are as desired. This proves the lemma.

We claim that the induction hypotheses (1) and (2) automatically pass up to $\alpha$ if $c f(\alpha)>\omega$. Namely, (1) holds at $\alpha$ by a $\Delta$-systenı argument. For (2), suppose that for an $\mathscr{F}_{w c}$-positive set $W$ of $\lambda$ 's there is a counterexample $\left\langle f_{\lambda}, g_{\lambda}, h_{\lambda}\right\rangle$. Let $N_{\lambda}=$ (support $f_{\lambda} \cup$ support $g_{\lambda}$ ). If $c f(\alpha) \neq \kappa$ then for some $\beta<\alpha$ and $\widetilde{F}_{w c}$-positive $V \subseteq W, \lambda \in V$ implies $N_{\lambda} \subseteq \beta$, and we are done. If $c f(\alpha)=\kappa$, pick a closed unbounded set $C \subseteq \kappa$ such that $\left\langle\sup \left\{\alpha_{\nu}: \nu\langle\lambda\}: \lambda \in C\right\rangle\right.$ is increasing, continuous and cofinal in $\alpha$ and an $\mathscr{F}_{w c}$-positive $V \subseteq W \cap C$ such that for some $\beta<\alpha$ and all $\lambda \in V, N_{\lambda} \cap \sup \left\{\alpha_{\nu}: \nu<\lambda\right\} \subseteq \beta$, then apply (2) at $\beta$.

Thus, we may assume for the rest of the proof that $\alpha$ is a successor ordinal or $c f(\alpha)=\omega$. Fix $\left\langle\mu_{n}: n\langle\omega\rangle\right.$ such that if $\alpha=\beta+1$ then each $\mu_{n}$ is the $\mu$ with $\alpha_{\mu}=\beta$, and if $c f(\alpha)=\omega$ then $\left\langle\alpha_{\mu_{n}}: n\langle\omega\rangle\right.$ is an increasing sequence converging to $\alpha$.

LEMMA 7. For $\mathscr{F}_{w c}$-almost all $\lambda$, the following holds: if $f, g \in \mathscr{P}_{\alpha}, h \in \mathscr{P}_{\alpha} \mid \lambda$ and $\#_{\lambda}^{\alpha}(f, g, h)$ then there exists $\left\langle f^{\prime}, g^{\prime}, h^{\prime}\right\rangle \geqslant\langle f, g, h\rangle$ such that $\#_{\lambda}^{\alpha}\left(f^{\prime}, g^{\prime}, h^{\prime}\right)$ and such that for each $\mu<\lambda$ with $\alpha_{\mu} \neq 0$, and each $\theta \in f^{\prime}\left(\alpha_{\mu}\right)-\lambda$, each $\tau \in g^{\prime}\left(\alpha_{\mu}\right)-\lambda$, $\left\langle f^{\prime} \uparrow \alpha_{\mu}, g^{\prime} \uparrow \alpha_{\mu}\right\rangle \lambda$-separates $\langle\theta, \tau\rangle$.

Proof. We prove the lemma for $\lambda$, assuming that $\lambda$ satisfies Lemmas 1 and 2, $\lambda>\mu_{n}(n<\omega)$ and for each $n<\omega, \lambda$ is in the $\mathscr{F}_{w c}$ set given by induction hypothesis (2) for $\alpha_{\mu_{n}}$. Construct $\left\langle f_{n}, g_{n}, h_{n}\right\rangle, n<\omega$, so that

(a) $f_{n}, g_{n} \in \mathscr{P}_{\alpha_{\mu_{n}}}, \#{ }_{\lambda}^{\alpha_{\mu_{n}}}\left(f_{n}, g_{n}, h_{n}\right)$,

(b) $\left\langle f \uparrow \alpha_{\mu_{n}} ; g \uparrow \alpha_{\mu_{n}}, h \uparrow \alpha_{\mu_{n}}\right\rangle \leqslant\left\langle f_{n}, g_{n}, h_{n}\right\rangle$,

(c) $\left\langle f_{n}, g_{n}, h_{n}\right\rangle \leqslant\left\langle f_{n+1}, g_{n+1}, h_{n+1}\right\rangle$,

(d) if, at stage $n>1,\left\langle\theta_{n}, \tau_{n}\right\rangle$ is the $n$th pair (in the appropriate bookkeeping list for exhausting them) with $\theta_{n} \in f_{n}\left(\alpha_{\nu_{n}}\right)-\lambda, \tau_{n} \in g_{n}\left(\alpha_{\nu_{n}}\right)-\lambda, \nu_{n}<\lambda, \alpha_{\nu_{n}} \leqslant \alpha_{\mu_{n}}$, then

$$
\left\langle f_{n} \uparrow \alpha_{\nu_{n}}, g_{n} \uparrow \alpha_{\nu_{n}}\right\rangle \lambda \text {-separates }\langle\theta, \tau\rangle \text {. }
$$

Let $f_{0}=f \uparrow \alpha_{\mu_{0}}, g_{0}=g \uparrow \alpha_{\mu_{0}}, h_{0}=h \uparrow \alpha_{\mu_{0}}$. Suppose $n>1$ and $f_{n-1}, g_{n-1}, h_{n-1}$ have been constructed. Let

$$
\begin{aligned}
& f_{n}^{\prime}=f_{n-1} \frown f \uparrow\left[\alpha_{\mu_{n-1}}, \alpha_{\mu_{n}}\right), \quad g_{n}^{\prime}=g_{n-1} \frown g \uparrow\left[\alpha_{\mu_{n-1}}, \alpha_{\mu_{n}}\right), \\
& h_{n}^{\prime}=h_{n-1} \frown h \uparrow\left[\alpha_{\mu_{n-1}}, \alpha_{\mu_{n}}\right) .
\end{aligned}
$$

Then $\#_{\lambda}^{\alpha_{\mu_{n}}}\left(f_{n}^{\prime}, g_{n}^{\prime}, h_{n}^{\prime}\right)$. By induction hypothesis (2), there is an $\bar{h}_{n} \geqslant h_{n}^{\prime}$ such that $*_{\lambda}^{\alpha_{\mu}}\left(f_{n}^{\prime}, g_{n}^{\prime}, \bar{h}_{n}\right)$. By Lemma 6 , there is $\left.\left\langle f_{n}^{\prime \prime}, g_{n}^{\prime \prime}, h_{n}^{\prime \prime}\right\rangle\right\rangle\left\langle f_{n}^{\prime}, g_{n}^{\prime}, \bar{h}_{n}\right\rangle$ such that $*_{\lambda}^{\alpha_{\mu_{n}}}\left(f_{n}^{\prime \prime}, g_{n}^{\prime \prime}, h_{n}^{\prime \prime}\right)$ and

$$
\left\langle f_{n}^{\prime \prime} \uparrow \alpha_{\nu_{n}}, g_{n}^{\prime \prime} \uparrow \alpha_{\nu_{n}}\right\rangle \text { separates }\left\langle\theta_{n}, \tau_{n}\right\rangle \text {. }
$$

Finally, by Lemma 5 we may choose $\left.\left\langle f_{n}, g_{n}, h_{n}\right\rangle\right\rangle\left\langle f_{n}^{\prime \prime}, g_{n}^{\prime \prime}, h_{n}^{\prime \prime}\right\rangle$ so that $\#{ }_{\lambda}^{\alpha_{\mu_{n}}}\left(f_{n}, g_{n}, h_{n}\right)$. 
Taking $f^{\prime}, g^{\prime}, h^{\prime}$ to be the coordinatewise unions of the $f_{n}$ 's, $g_{n}$ 's, $h_{n}$ 's gives the lemma.

We now verify the two induction hypotheses.

(1) $\mathscr{P}_{\alpha}$ has the $\kappa c c$.

Proof. Given $f_{\lambda} \in \mathscr{P}_{\alpha}, \lambda<\kappa$. For each $\lambda$ which satisfies Lemmas 1, 2 and 7, with $\lambda>\mu_{n}(n<\omega)$, apply Lemma 7 to the triple $\left\langle f_{\lambda}, f_{\lambda}, f_{\lambda} \mid \lambda\right\rangle$, obtaining a triple $\left\langle f_{\lambda}^{*}, f_{\lambda}^{* *}, j_{\lambda}\right\rangle\left(\right.$ so $\left.f_{\lambda} \leqslant f_{\lambda}^{*}, f_{\lambda}^{* *}, j_{\lambda}=f_{\lambda}^{*}\left|\lambda=f_{\lambda}^{* *}\right| \lambda\right)$.

Let

$$
B_{\lambda}=\left(\text { support } f_{\lambda}^{*} \cup \operatorname{support} f_{\lambda}^{* *}\right) \cap\left\{\alpha_{\mu}: \mu<\lambda\right\} .
$$

If $0 \neq \alpha_{\mu} \in B_{\lambda}$, write

$$
\begin{aligned}
f_{\lambda}^{*}\left(\alpha_{\mu}\right)-\lambda=\left\{\theta_{\mu \lambda n}: n<r_{\mu \lambda}\right\}, & r_{\mu \lambda} \leqslant \omega, \\
f_{\lambda}^{* *}\left(\alpha_{\mu}\right)-\lambda=\left\{\tau_{\mu \lambda m}: m<s_{\mu \lambda}\right\}, & s_{\mu \lambda} \leqslant \omega .
\end{aligned}
$$

To each pair $\left\langle\theta_{\mu \lambda n}, \tau_{\mu \lambda m}\right\rangle, n<r_{\mu \lambda}, m<s_{\mu \lambda},\left\langle f_{\lambda}^{*}, f_{\lambda}^{* *}\right\rangle$ assigns a separating pair $\left\langle\theta_{\mu \lambda n}^{\prime}, \tau_{\mu \lambda m}^{\prime}\right\rangle \in \lambda \times \lambda$.

Let $J_{\lambda}=\left(\operatorname{dom} f_{\lambda}^{*}(0) \cup \operatorname{dom} f_{\lambda}^{* *}(0)\right)-\left(\omega_{1} \times \lambda\right)$.

By the normality of $\mathscr{F}_{w c}$, there is an $\mathscr{F}_{w c}$-positive set $U$ such that on $U$, the sets $B_{\lambda}, r_{\mu \lambda}, s_{\mu \lambda}, \theta_{\mu \lambda n}^{\prime}, \tau_{\mu \lambda m}^{\prime}, J_{\lambda}$ are independent of $\lambda$, and such that if $\lambda, \lambda^{\prime} \in U, \lambda<\lambda^{\prime}$, then (support $f_{\lambda}^{*} \cup$ support $f_{\lambda}^{* *}$ ) $\cap$ (support $f_{\lambda^{\prime}}^{*} \cup$ support $f_{\lambda^{\prime}}^{* *}$ ) $=B_{\lambda}$, and $J_{\lambda} \cap J_{\lambda^{\prime}}$ $=\varnothing$.

By induction on $\gamma \leqslant \alpha$ it is seen that if $\lambda, \mu \in U$ and $\lambda<\mu$, then $f_{\lambda}^{*} \sim f_{\mu}^{* *}$. Namely, there is no trouble with coordinates in the support of at most one of these functions; coordinates in both supports, being in $B_{\lambda}$, are taken care of by the construction. Since $f_{\lambda} \leqslant f_{\lambda}^{*}$ and $f_{\mu} \leqslant f_{\mu}^{* *}$, we are done.

The following strengthening of $\kappa c c$ for $P_{\alpha}$ has thus been proved: if for an $\mathscr{F}_{w c}$-positive set $W$ of $\lambda$ 's, \# ${ }_{\lambda}^{\alpha}\left(f_{\lambda}, g_{\lambda}, h_{\lambda}\right)$, then there is an $\mathscr{F}_{w c}$-positive $U \subseteq W$ and $\left(f_{\lambda}^{\prime}, g_{\lambda}^{\prime}, h_{\lambda}^{\prime}\right), \lambda \in U$, such that $\left\langle f_{\lambda}, g_{\lambda}, h_{\lambda}\right\rangle \leqslant\left\langle f_{\lambda}^{\prime}, g_{\lambda}^{\prime}, h_{\lambda}^{\prime}\right\rangle, \#_{\lambda}^{\alpha}\left(f_{\lambda}^{\prime}, g_{\lambda}^{\prime}, h_{\lambda}^{\prime}\right)$, and so that if $\lambda, \mu \in W, \lambda<\mu$, then $f_{\lambda}^{\prime} \sim g_{\mu}^{\prime}$ in the strong sense that the coordinatewise union of $f_{\lambda}^{\prime}$ and $g_{\mu}^{\prime}$ is a condition extending both $f_{\lambda}^{\prime}$ and $g_{\mu}^{\prime}$.

Lastly, we prove the second induction hypothesis for $\alpha$.

(2) For $\mathscr{F}_{w c}$-almost all $\lambda<\kappa$, for all $f, g, h$, \# ${ }_{\lambda}^{\alpha}(f, g, h)$ implies that for some $h^{\prime} \geqslant h, *_{\lambda}^{\alpha}\left(f, g, h^{\prime}\right)$.

Proof. Otherwise for an $\mathscr{F}_{w c}$-positive set $W$ of $\lambda$ 's there exists a counterexample $\left\langle f_{\lambda}, g_{\lambda}, h_{\lambda}\right\rangle$. We may assume that for each $\lambda \in W, \lambda>\mu_{n}(n<\omega)$ and $\lambda$ satisfies Lemmas 1, 2 and 7. Furthermore, since we have already proved that $\mathscr{P}_{\alpha}$ has the $\kappa c c$, we may assume that for each $\lambda \in W, \mathscr{P}_{\alpha} \mid \lambda \subseteq_{\text {reg }} \mathscr{P}_{\alpha}$ and $\mathscr{P}_{\alpha} \mid \lambda$ has the $\lambda c c$. If $f_{\lambda}$ or $g_{\lambda}$ equals $h_{\lambda}$ we are done, so assume, for each $\lambda \in W$, that $f_{\lambda}, g_{\lambda} \notin \mathscr{P}_{\alpha} \mid \lambda$.

Apply Lemma 7 to each $\left\langle f_{\lambda}, g_{\lambda}, h_{\lambda}\right\rangle, \lambda \in W$, getting $\left\langle f_{\lambda}^{\prime}, g_{\lambda}^{\prime}, h_{\lambda}^{\prime}\right\rangle$. Now uniformize as in part (a) to get an $\mathscr{F}_{w c}$-positive $V \subseteq W$ such that if $\lambda, \mu \in V$ and $\lambda<\mu$ then $f_{\lambda}^{\prime} \sim g_{\mu}^{\prime}$. Since $\left\langle f_{\lambda}, g_{\lambda}, h_{\lambda}\right\rangle$ is a counterexample to (b), there is a maximal antichain $H_{\lambda}$ of $\left\{h \in \mathscr{P}_{\alpha} \mid \lambda: h \geqslant h_{\lambda}\right\}$ such that for each $h \in H_{\lambda}, h \nsim f_{\lambda}$ or $h \nsim g_{\lambda}$. Then $H_{\lambda}$ is a maximal antichain of $\left\{h \in \mathscr{P}_{\alpha}: h \geqslant h_{\lambda}\right\}$, and Card $H_{\lambda}<\lambda$. Pick an $\mathscr{F}_{w c}$-positive $U \subseteq V$ on which $H_{\lambda}=H$ is independent of $\lambda$ and such that for each $h \in H$, the questions, whether or not $h \sim f_{\lambda}, h \sim g_{\lambda}$, are independent of $\lambda$. Pick 
$\lambda, \mu \in U, \lambda<\mu$, and let $j \geqslant f_{\lambda}^{\prime}, g_{\mu}^{\prime}$. Now $j \geqslant h_{\lambda}$, and $j \notin \mathscr{P}_{\alpha} \mid \lambda$ (whence $j \notin H$ ). But for each $h \in H$, either $h \nsim g_{\lambda}$ (whence $h \nsim g_{\mu}$ ) or $h \nsim f_{\lambda}$. In either case, $h \nsim j$ since $j \geqslant f_{\lambda}, g_{\mu}$, so $H$ is not maximal, a contradiction.

This completes the proof of the theorem.

Denote by an $\omega_{2}$-tree a tree $T$ of any cardinality with no paths of length $\omega_{2}$. An $\omega_{2}$-tree $T$ is special if there is an $f: T \rightarrow \omega_{1}$ such that $x<_{T} y$ implies $f(x) \neq f(y)$. By the previous methods, using countable specializing functions instead of countable antichains, the consistency of " $2^{\kappa_{0}}=\kappa_{1}, 2^{\kappa_{1}}>\kappa_{2}$, and every $\omega_{2}$-tree of cardinality $<2^{\kappa_{1}}$ is special" is obtained - the analogous theorem for the $\kappa_{1}$ case being Baumgartner-Malitz-Reinhardt [1]. We can also get this model to satisfy the "generalized Martin's axioms" (which are consistent relative to just ZFC but which do not imply $\mathrm{SH}_{\mathrm{N}_{2}}$ ) that have been considered by the first author and by Baumgartner (see Tall [8]). Desirable, of course, would be the consistency of a generalized $M A$ which is both simple and powerful.

The partial orderings appropriate for the prior methods can be iterated an arbitrary number of times, giving generalized $M A$ models in which $2^{N_{1}}$ is arbitrarily large. The ordering $\mathscr{R}_{\alpha}$ giving the first $\alpha$ steps of the iteration need not be of cardinality $\leqslant \kappa$, but, assuming each $\mathscr{R}_{\beta}, \beta\left\langle\alpha\right.$, has $\kappa c c$, any sequence $\left\langle p_{\lambda}: \lambda\langle\kappa\rangle\right.$ from $\Re_{\alpha}$ is a subset of a sufficiently closed model of power $\kappa$, in which the proof that two $p_{\lambda}$ 's are compatible can be carried out.

Regarding the analog of these results where $\kappa_{2}$ is replaced by $\gamma^{+}$-the relevant forcing is $\gamma$-directed closed, so by upward Easton forcing we may guarantee that, for example, $\gamma$ remains supercompact if it was in the ground model.

For results involving consequences of $\mathrm{SH}_{\mathrm{k}_{2}}$ : with $\mathrm{GCH}$, see Gregory [3], [4] (Con $\left(\mathrm{SH}_{\mathrm{K}_{2}}\right.$ and $\left.\mathrm{GCH}\right)$ is open); with just $\mathrm{CH}$, see a forthcoming paper by Stanley and the second author.

\section{REFERENCES}

0. J. Baumgartner, Ineffability properties of cardinals. I, Proc. Colloq. Infinite and Finite Sets, Bolyai Janos Society, Hungary, 1975, pp. 109-130.

1. J. Baumgartner, J. Malitz and W. Reinhardt, Embedding trees in the rationals, Proc. Nat. Acad. Sci. U.S.A. 67 (1970), 1748-1755.

2. K. Devlin, handwritten notes.

3. J. Gregory, Higher Souslin trees and the generalized continuum hypothesis, J. Symbolic Logic 41 (1976), 663-671.

4. R. Jensen, The fine structure of the constructible hierarchy, Ann. Math. Logic 4 (1972), 229-308.

5. W. Mitchell, Aronszajn trees and the independence of the transfer property, Ann. Math. Logic 5 (1973), 21-46.

6. S. Shelah, A weak generalization of MA to higher cardinals, Israel J. Math. 30 (1978), 297-306.

7. R. Solovay and S. Tennenbaum, Iterated Cohen extensions and Souslin's problem, Ann. of Math. (2) 94 (1971), 201-245.

8. F. Tall, Some applications of a generalized Martin's axiom.

9. A. Levy, The sizes of the indescribable cardinals, Proc. Sympos. Pure Math., vol. 13, Amer. Math. Soc., Providence, R.I., 1971, pp. 205-218.

Department of Mathematics, University of Colorado, Boulder, Colorado 80309

Department of Mathematics, Hebrew University, Jerusalem, IsRael 